\title{
Observations of Type Ia Supernovae and Challenges for Cosmology
}

\author{
Weidong Li and Alexei V. Filippenko \\ Department of Astronomy, University of California, Berkeley, CA 94720-3411, \\ USA; \\ wli@astro.berkeley.edu, alex@astro.berkeley.edu
}

Summary. Observations of Type Ia supernovae (SNe Ia) reveal correlations between their luminosities and light-curve shapes, and between their spectral sequence and photometric sequence. Assuming SNe Ia do not evolve at different redshifts, the Hubble diagram of SNe Ia may indicate an accelerating Universe, the signature of a cosmological constant or other forms of dark energy. Several studies raise concerns about the evolution of SNe Ia (e.g., the peculiarity rate, the rise time, and the color of SNe Ia at different redshifts), but all these studies suffer from the difficulties of obtaining high-quality spectroscopy and photometry for SNe Ia at high redshifts. There are also some troubling cases of SNe Ia that provide counterexamples to the observed correlations, suggesting that a secondary parameter is necessary to describe the whole SN Ia family. Understanding SNe Ia both observationally and theoretically will be the key to boosting confidence in the SN Ia cosmological results.

\section{Observations of SNe Ia and the Accelerating Universe}

Spectroscopic observations of nearby Type Ia supernovae ( $\mathrm{SNe}$ Ia) reveal that they can be divided into several subclasses: the majority are the so-called "normal" or "Branch normal" SNe Ia [6], while the others are "peculiar" SNe Ia which can be further divided into SN 1991T-like or SN 1991bg-like objects (see [11] and references therein). Li et al. [27] discuss SN 1999aa-like objects as another potential subclass of the peculiar SNe Ia. The classification is based on the spectra of SNe Ia before or near maximum light: normal $\mathrm{SNe}$ Ia show conspicuous features of Si II, Ca II, and other intermediatemass elements (IMEs; e.g., S II, O I); SN 1991T-like objects show unusually weak IME lines, yet prominent high-excitation features of Fe III; SN $1991 \mathrm{bg}-$ like objects have strong IME features, plus a broad Ti II absorption trough around $4100 \AA$ and enhanced Si II/Ti II $\lambda 5800$ absorptions. SN 1999aa-like objects are similar to the SN 1991T-like ones, but with significant Ca II H \& $\mathrm{K}$ absorption lines.

Photometric observations of nearby SNe Ia also reveal a correlation between the peak luminosity and light-curve shape (LLCS correlation, hereafter). This was first convincingly demonstrated by Phillips (1993), and subsequently exploited by $[16,34,37,38]$. The slower, broader light curves are intrinsically brighter at peak than the faster, narrower light curves. Various 
parameters have been proposed to quantify the "speed" of the light curve, such as $\Delta m_{15}(B)$ (the decline in magnitudes between peak brightness and 15 days later in the $B$ band), $\Delta$ (the difference in magnitudes between the peak brightness of a SN Ia and a nominal standard SN Ia), $s$ ("stretch factor," the amount of stretch applied to the light curve (generally $B$ band) of a SN Ia to match those of a nominal standard SN Ia), and various empirical methods have been developed to calibrate the peak absolute magnitudes of $\mathrm{SNe} \mathrm{Ia}[16,23,34,36,38,51]$. The multi-color light-curve shape (MLCS) method, for example, has demonstrated the ability to achieve a scatter in the calibrated absolute magnitudes of SNe Ia to $\sim 0.15 \mathrm{mag}$ [38].

By assuming that the observed correlation for nearby SNe Ia also applies to the objects at high redshift, utilizing the empirical calibration methods developed from nearby SNe Ia, and studying the Hubble diagram for SNe Ia at both low and high redshifts, the High-z SN Search Team [45] and the SN Cosmology Project [34] have measured that high-redshift SNe Ia are fainter than expected, and interpreted this result as evidence that the expansion of the Universe is accelerating, due perhaps to a non-zero cosmological constant or some other forms of dark energy (e.g., $[24,35,39,47]$.

\section{Challenges of the Accelerating Universe}

The observational fact is that the SNe Ia at $z \approx 0.5$ are $\sim 0.28$ mag dimmer ( $\sim 14 \%$ farther) than expected in a Universe with $\Omega_{M}=0.3$ and no cosmological constant. Besides the interpretation of a positive cosmological constant, other possible alternatives have been proposed as follows.

1. Luminosity evolution. Two important questions are (a) whether the highredshift SNe Ia follow the same LLCS correlation for the nearby objects, and (b) whether the SNe Ia at $z \approx 0.5$ are intrinsically fainter than nearby SNe Ia by $0.28 \mathrm{mag}$ after correction for the LLCS correlation.

2. Interstellar dust, which produces more extinction of the high-redshift SNe Ia and makes them look apparently fainter.

3. Selection bias: preferentially fainter SNe Ia are observed at high redshift.

4. Gravitational lensing: the inhomogeneous distribution of matter in the Universe deamplifies the observed brightness of most high-redshift SNe Ia.

Among these, ordinary dust is not considered a viable option, as it introduces too much reddening in the colors of SNe Ia at high redshift [41, 42] and more dispersion in the distance measurements than is currently observed. "Grey" dust [1,2], which leaves little or no imprint on the spectral energy distribution of a SN Ia, could be more pernicious, but the amount of grey dust required to explain the faintness of high-redshift $\mathrm{SNe}$ Ia would also distort the cosmic microwave background, an effect which has not been seen. Moreover, it still introduces more distance dispersion than is currently observed. 
Tonry et al. [47] also provided evidence that a systematic effect which goes as a power law of $(1+z)$, such as extinction by dust, is not likely to match the SN Ia data. No known selection bias favors detection of intrinsically fainter SNe Ia at high redshift. Malmquist bias may afflict the nearby SN Ia sample so preferentially brighter objects were observed, but the effect is shown to be small $[35,39]$. Moreover, the high-redshift SN Ia sample should be subject to the same bias to a greater extent, since most high-redshift SN searches are magnitude-limited. Gravitational lensing deamplification [4, 32], typically $2 \%$ at $z=0.5$, is much smaller than the cosmological effect.

Luminosity evolution is arguably the most serious challenge to the cosmological interpretation of high-redshift SNe Ia. The cause of this is somewhat embarrassing: despite being the most luminous type of SN, SNe Ia have not been completely understood theoretically (see [25] for a review), or have the progenitor system conclusively identified (see [30] for a review). Consequently, theory cannot provide conclusive guidance on whether or how SNe Ia and their progenitor systems evolve at different redshifts. Nevertheless, theorists have provided some insights into this question by studying the effects of metallicity and the $\mathrm{C} / \mathrm{O}$ ratio of a white dwarf (WD), the agreed precursor to a SN Ia (e.g., [22, 33, 48, 49]). Höflich, Wheeler, \& Thielemann [22] suggested that the effect of changing metallicity on the rest-frame visual and blue light curves is small, and as the $\mathrm{C} / \mathrm{O}$ ratio of a WD becomes progressively lower at higher redshift, the luminosity of the resulting SN Ia becomes brighter for the same light-curve shape, an effect that is contrary to the cosmological result from SNe Ia. Umeda et al. [48] and Nomoto et al. [33] use the variation in the $\mathrm{C} / \mathrm{O}$ ratio in the $\mathrm{WD}$ to explain the distribution of $\mathrm{SN}$ Ia brightness, but suggest that the diversity can be normalized by applying the LLCS correlation.

Answers to the question of whether SNe Ia evolve have also been sought from observations of them at different redshifts. The nearby sample is an excellent laboratory for studying possible luminosity evolution, since SNe Ia have been observed in a wide range of host-galaxy morphologies including ellipticals, spirals, irregulars, and dwarf galaxies. In fact, the range of metallicity, stellar age, and interstellar environments probed by the nearby SN Ia sample is much greater than the mean evolution in these properties for individual galaxies between $z=0$ and $z=0.5$. Some variation of the observed characteristics of SNe Ia has been noticed; for example, luminous events occur preferentially in metal-poor environments [19], and the luminosity of SNe Ia correlates with the projected distance from the host nucleus [50]. However, after correction for the LLCS correlation and extinction, the observed residuals from the Hubble flow do not correlate with host-galaxy morphology or the projected radial distances $[41,42,46]$. This suggests that the LLCS correlation applies to a wide range of stellar environments and is a strong argument against significant evolution to $z=0.5[45]$. 
The empirical test of luminosity evolution at high redshift has been focused on getting high-quality spectra and light curves, and comparing them with those of nearby SNe Ia. The assumption of this test is that significant luminosity evolution would be accompanied by other visibly altered observables of the SNe. Comparison of high-quality spectra between nearby and high-redshift SN Ia have revealed remarkable similarity [8, 35, 39, 47]. Riess et al. [41, 42] also obtained the rest-frame $I$-band light curve of the highredshift SN Ia 1999Q, which displayed the secondary maximum that is typical of normal nearby SNe Ia.

To date, there is no clear, direct evidence that suggests significant luminosity evolution for SNe Ia at different redshift. To rest the case of luminosity evolution, however, we need to fully understand the models of SNe Ia and how they evolve at different redshifts. Without a firm theoretical footing, we must conservatively demand that all observables of high-redshift SNe Ia be statistically consistent with their nearby counterparts.

In the following sections, we discuss in more detail some of the recent comparisons done on the observables and characteristics of SNe Ia at different redshifts, which mag suggest possible differences between high-redshift SNe Ia and their nearby counterparts.

\subsection{Peculiarity Rate at Different Redshifts}

The rate of "peculiar SNe Ia" in the nearby sample has been recently measured by [27]. They used a distance-limited sample of SNe Ia from the Lick Observatory Supernova Search (LOSS; [12]) and performed Monte Carlo simulations [26] demonstrating that essentially all SNe Ia should have been discovered in the sample galaxies of LOSS. Within this unbiased sample they found a rate of $\sim 20 \%$ for the SN 1991T/1999aa-like objects and $\sim 16 \%$ for the SN $1991 \mathrm{bg}$-like objects, for a total peculiarity rate of $\sim 36 \%$.

However, in the now more than 100 spectroscopically classified SNe Ia at high redshift, there has not been a single unambiguously peculiar SN Ia reported. While the lack of SN 1991bg-like objects could be explained by their intrinsic faintness and low expected rate in magnitude-limited searches, the lack of SN 1991T/1999aa-like objects is puzzling.

Li et al. [27] offered several possible explanations for the difference between the peculiarity rate of SNe Ia at different redshifts: extinction toward the SN 1991T/1999aa-like objects, difficulty in identifying peculiarities in poor-quality spectra of the high-redshift $\mathrm{SNe}$ Ia, and most importantly, the "age bias": the peculiarity of SN 1991T/1999aa-like objects can only be easily identified in early-time spectra. This same bias may also explain why the Calán/Tololo survey, a nearby magnitude-limited SN search, yielded no SN 1991T/1999aa-like objects among 29 SNe Ia [17].

If, however, these observational biases are not to blame, the absence of peculiar SNe Ia at high redshift could result from an evolution of the population of progenitor systems: certain progenitor channels at high redshift 
may be lost due to a redshift-dependent variation in the mass, composition, and metallicity of SN Ia progenitors (e.g., $[30,44]$ ). It is thus an important challenge to observationally identify some peculiar SNe Ia at high redshifts, to definitively rule out luminosity evolution as the cause of the difference in the peculiarity rate of $\mathrm{SNe} I \mathrm{I}$ at different redshifts.

\subsection{Rise Time at Different Redshifts}

The rise time is defined as the time interval between the explosion and the maximum brightness of a SN Ia. Precise knowledge of the SN Ia rise time, which is sensitive to the ejecta opacity and the distribution of ${ }^{56} \mathrm{Ni}$, provides constraints on models of SN Ia progenitors. A comparison of the rise time for high-redshift and nearby SNe Ia is thus a valuable test of luminosity evolution.

The rise time for the nearby SNe Ia was measured by [40]. They collected about 25 measurements of SNe Ia between 10 and 18 days before $B$ maximum, normalized them to a fiducial rise time curve, and measured a rise time of $19.98 \pm 0.15$ days. A preliminary rise time for the high-redshift SNe Ia was measured by [15] from the Supernova Cosmological Project (SCP) data as $17.50 \pm 0.40$ days, which is discrepant from the nearby rise time at a statistical likelihood greater than $99.99 \%$ (5.8\%). Aldering, Knop, \& Nugent [3], however, refined the rise time for the high-redshift SNe Ia in the SCP data to $17.50 \pm 1.20$ days, $\mathrm{a} \sim 2 \sigma$ difference from the nearby measurement. They also suggested that under extreme situations the rise time could be biased up to 2-3 days due to observational biases and fitting methods.

It remains to be seen whether the rise times of high-redshift and nearby SNe Ia are statistically inconsistent when a better rise time measurement is derived for the high-redshift SNe Ia. It should also be noted that even if the two rise times are inconsistent with each other, it is unclear whether the difference in rise time could be translated into a difference in peak luminosity: most current theoretical models have difficulties in reproducing the observed rise times and the correlation between rise time and peak luminosity.

\subsection{Intrinsic Color at Different Redshifts}

To date, there is no consensus on the precise intrinsic colors of SNe Ia with different photometric behaviors. As a result, there is not a good theoretical or empirical method to accurately determine host-galaxy extinction to $\mathrm{SNe} \mathrm{Ia}$, and observers often have to resort to priors such as applying the Galactic reddening law to the host galaxies of SNe Ia, and assuming all SNe Ia have the same intrinsic color at maximum. Phillips et al. [37] proposed a method to estimate the host-galaxy reddening to a SN Ia by using its color at 3060 days past maximum, but [28] showed that this method does not apply to all objects. The extinction correction is a major source of uncertainty in 
the current empirical fitting methods. To circumvent this difficulty, people often use subsamples that are likely to have low extinction at both low and high redshifts. Fortunately, [21] showed that most SNe Ia should have low extinction.

Different methods for treating the extinction correction in the fitting process yield different results in the intrinsic color comparison of SNe Ia at a range of redshifts. Leibundgut [25] and Falco et al. [10] suggested that there is evidence from the $E(B-V)$ values in [39] that high-redshift SNe Ia are statistically bluer than their nearby counterparts, but analysis by [35] showed no such effect, nor did a recent compilation of 11 high-redshift SNe Ia observed by the Hubble Space Telescope [24]. The problem of extinction correction will continue to plague the empirical fitting methods until a better understanding of the intrinsic colors of SNe Ia is achieved, and the influence of extinction corrections on the cosmological conclusions needs to be investigated in more detail. Drell, Loredo, \& Wasserman [9], for example, attributed the difference in colors to luminosity evolution, while [24] suggested that reasonable changes in colors do not have a significant impact on the cosmological results.

\subsection{Peculiar Nearby SNe Ia}

A fundamental assumption of the current empirical fitting methods is that the light curves of all normal SNe Ia can be represented by a single parameter such as $\Delta m_{15}(B), \Delta$, or $s$. However, there is growing evidence that not all SNe Ia form a one-parameter family. Branch [5] showed that normal SNe Ia could have very different expansion velocities. Hamuy et al. [18] showed that some light curves with similar decline rates have significant differences in particular details. Hatano et al. [21] also demonstrated that the spectroscopic diversity among $\mathrm{SNe}$ Ia is multi-dimensional.

The SN 1991T-like and SN 1991bg-like objects, though categorized as peculiar SNe Ia, generally follow the LLCS correlation and the spectrumluminosity sequence - i.e., they seem to be an extension of the one-parameter description of normal SNe Ia. Some more disturbing cases of peculiar nearby SNe Ia that fail the one-parameter description are SN 2000cx [7, 26], SN 2002cx [29], SN 2001ay (Nugent et al. in preparation), and SN 2002ic [20].

The peculiarity of SN $2000 \mathrm{cx}$ is that its light curves cannot be fit well by the existing fitting methods. There is an apparent asymmetry in the $B$ band peak, in which the premaximum brightening is relatively fast (similar to that of the normal SN 1994D), but the postmaximum decline is relatively slow (similar to that of the overluminous SN 1991T). SN 2000cx has very blue colors and also unique spectral evolution. Its premaximum spectra are similar to those of SN 1991T-like objects, but the high-excitation Fe III lines remain prominent until well after maximum. The expansion velocities derived from the absorption features are unusually high and evolve differently than normal. Though it has a slow light curve, its estimated luminosity is average [26] or even slightly subluminous [7]. 
SN 2002cx has many properties that are the opposite of those of SN $2000 \mathrm{cx}$. It has a premaximum spectrum similar to that of SN 1991T, a decline rate in the $B$-band similar to that of normal $\mathrm{SNe} \mathrm{Ia}$, but a luminosity similar to that of the very subluminous SN 1991bg. It has a very red color evolution, and has extremely low expansion velocities measured from spectral features. The $R$ and $I$-band light curves have a peculiar plateau phase around maximum. The late-time decline rate in all $B V R I$ bands is unusually slow. SN 2003gq [13] may be another event that is similar to SN 2002cx.

SN 2001ay has a normal near-maximum spectrum, except that it has very high expansion velocities. The light curves of SN 2001ay are the slowest ever recorded, yet it has a normal luminosity. It also has a peculiar red color evolution until 30 days after maximum.

SN 2002ic is the only SN Ia to have shown direct evidence of SN ejecta interacting with the circumstellar medium (CSM). Its near-maximum spectrum is similar to that of SN 1991T, but diluted in strength. There are remarkable Balmer lines in later spectra, with $H \alpha$ showing an unresolved component (FWHM $<300 \mathrm{~km} \mathrm{~s}^{-1}$ ) superimposed on a broad resolved base (FWHM $\approx 1800 \mathrm{~km} \mathrm{~s}^{-1}$ ), similar to those observed in Type IIn SNe [11]. The spectral features and photometric behavior of SN 2002ic suggest that it has a very dense CSM. Based on these observations, [20] ruled out the doubledegenerate model for SN 2002ic, and suggested that the progenitor system involves a white dwarf and an asymptotic giant branch star. Livio \& Riess [31], however, argued that the opposite may be true: SN 2002ic results from a rare circumstance in which the SN Ia ejecta interact with the previously ejected common envelope of a double-degenerate system.

Although the frequency of these peculiar SNe Ia is low, and statistically they will not challenge the established empirical correlations, we need to understand why they are peculiar, and how they can be fit into the whole picture of SN Ia theories and observations. These objects with unusual properties might represent the general models of SNe Ia under extreme conditions, and studying them will provide clues to the theoretical models and progenitor systems. It is interesting to note that three of the four peculiar SNe Ia (SNe 2000cx, 2002cx, and 2001ay) all have very unusual expansion velocities, and three (SNe 2000cx, 2002cx, and 2002ic) show spectral features similar to those of SN 1991T. The subclass of SN 1991T/1999aa-like objects may thus be more heterogeneous than other SNe Ia, and objects with unusual expansion velocities should be treated with caution when used as cosmological tools.

\section{Conclusions}

Many alternatives have been proposed to explain the SN Ia data at different redshifts, but so far none has seriously challenged the accelerating Universe 
result. We have found no clear, direct evidence that SNe Ia at different redshifts evolve, though some studies show that there may exist some differences in their peculiarity rate, rise time, or colors.

The key to boosting confidence in the cosmological results from SNe Ia is to understand SNe Ia both theoretically and observationally. We need to theoretically identify the elusive progenitor systems for $\mathrm{SNe} \mathrm{Ia}$, and find out the cause of the diversity of SNe Ia. Similarly, we need to continue to search for SN/CSM interactions such as that observed in SN 2002ic, and place stringent constraints on the accretion history of SN Ia progenitors. We also need to re-examine existing observations of $\mathrm{SNe}$ IIn, to investigate whether SN 2002ic is an isolated case, or whether some additional SNe IIn are actually SNe Ia with strong SN/CSM interaction. For nearby SNe Ia, we need to develop better methods to measure host-galaxy extinction than currently available, study the environmental effects, find more empirical correlations, and develop a subclassification scheme that possibly links to different progenitor channels. We should continue to study those SNe Ia that are clearly discrepant. For high-redshift $\mathrm{SNe} \mathrm{Ia}$, we need to identify some peculiar SN 1991T-like or SN 1991bg-like objects, get better rise time measurements, obtain more high-quality spectra and light curves, and compare them with those of nearby SNe Ia. The ESSENCE project (e.g., [14]), SNAP satellite (http://snap.lbl.gov/), and the higher-z project [43] are prime examples of current and future extensive studies of high-redshift SNe Ia.

Acknowledgement. Our research is currently supported by NSF grants AST-0206329 and AST-0307894, by the Sylvia \& Jim Katzman Foundation, and by NASA grants GO-8641, GO-9114, and GO-9352 from the Space Telescope Science Institute, which is operated by AURA, Inc., under NASA contract NAS 5-26555. We thank the conference organizers for partial travel funds.

\section{References}

1. A.N. Aguirre: Astrophys. J. Lett. 512, L19 (1999)

2. A.N. Aguirre: Astrophys. J. 525, 583 (1999)

3. G. Aldering, R. Knop, P. Nugent: Astron. J. 119, 2110 (2000)

4. A.J. Barber: Mon. Not. R. Astron. Soc. 318, 195 (2000)

5. D. Branch: Astrophys. J. Lett. 316, L81 (1987)

6. D. Branch, A. Fisher, P. Nugent: Astron. J. 106, 2383 (1993)

7. P. Candia et al. : Pub. Astron. Soc. Pacific 115, 277 (2003)

8. A. Coil et al. : Astrophys. J. Lett. 544, L111 (2000)

9. P.S. Drell, T.J. Loredo, I. Wasserman: Astrophys. J. 530, 593 (2000)

10. E. Falco et al. : Astrophys. J. 523, 617 (1999)

11. A.V. Filippenko: Ann. Rev. Astron. Astrophys. 35, 309 (1997)

12. A.V. Filippenko, W.D. Li, R.R. Treffers, M. Modjaz: In: Small-Telescope Astronomy on Global Scales, eds. W.P. Chen, C. Lemme, B. Paczyński (San Francisco: Astron. Soc. Pac., 2001) p. 121 
13. A.V. Filippenko, R. Foley, R.: IAUC (2003)

14. P.M. Garnavich et al. : Bull. Am. Astron. Soc. 201, 7809 (2002)

15. G. Goldhaber: Bull. Am. Astron. Soc. 193, 4713 (1998)

16. M. Hamuy et al. : Astron. J. 112, 2391 (1996)

17. M. Hamuy et al. : Astron. J. 112, 2408 (1996)

18. M. Hamuy et al. : Astron. J. 112, 2438 (1996)

19. M. Hamuy et al. : Astron. J. 120, 1479 (2000)

20. M. Hamuy et al. : Nature 424651 (2003)

21. K. Hatano, D. Branch, J. Deaton: Astrophys. J. 502, 177 (1998)

22. P. Höflich, J.C. Wheeler, F.-K. Thielemann: Astrophys. J. 502, 177 (1998)

23. S. Jha: PhD thesis, Harvard Univ. (2002)

24. R.A. Knop et al. : astro-ph 0309368

25. B. Leibundgut: Ann. Rev. Astron. Astrophys. 39, 67 (2001)

26. W. Li, A.V. Filippenko, A.G. Riess: Astrophys. J. 546, 719 (2001)

27. W. Li et al. : Astrophys. J. 546, 734 (2001)

28. W. Li et al. : Pub. Astron. Soc. Pacific 113, 1178 (2001)

29. W. Li et al. : Pub. Astron. Soc. Pacific 115, 453 (2003)

30. M. Livio: In: Type Ia Supernovae, Theory and Cosmology, eds. J.C. Niemeyer, J.W. Truran (Cambridge University Press, 2000) p. 33

31. M. Livio, A.G. Riess: Astrophys. J. Lett. 594, L93 (2003)

32. R.B. Metcalf: Mon. Not. R. Astron. Soc. 305, 746 (1999)

33. K. Nomoto et al. : In: From Twilight to Highlight: The Physics of Supernovae, eds. W. Hillebrandt, B. Leibundgut (Berlin: Springer, 2003) p. 115

34. S. Perlmutter et al. : Astrophys. J. 483, 565 (1997)

35. S. Perlmutter et al. : Astrophys. J. 517, 565 (1999)

36. M.M. Phillips: Astrophys. J. Lett. 413, L105 (1993)

37. M.M. Phillips et al. : Astron. J. 118, 1766 (1999)

38. A.G. Riess, W.H. Press, R.P. Kirshner: Astrophys. J. 473, 88 (1996)

39. A.G. Riess et al. : Astron. J. 116, 1009 (1998)

40. A.G. Riess et al. : Astron. J. 118, 2675 (1999)

41. A.G. Riess: Pub. Astron. Soc. Pacific 112, 1284 (2000)

42. A.G. Riess et al. : Astrophys. J. 536, 62 (2000)

43. A.G. Riess: Bull. Am. Astron. Soc. 201, 3901 (2002)

44. P. Ruiz-Lapuente, R. Canal: Astrophys. J. 497, 57 (1998)

45. B.P. Schmidt et al. : Astrophys. J. 507, 46 (1998)

46. M. Sullivan et al. : Mon. Not. R. Astron. Soc. 340, 1057 (2003)

47. J.L. Tonry et al. : Astrophys. J. 594, 1 (2003)

48. H. Umeda et al. : Astrophys. J. 522, 43 (1999)

49. T. von Hippel, G.D. Bothun, R.A. Schommer: Astron. J. 114, 1154 (1997)

50. L. Wang, P. Höflich, J.C. Wheeler: Astrophys. J. 483, 29 (1997)

51. L. Wang, G. Goldhaber, G. Aldering, S. Perlmutter: Astrophys. J. 590, 944 (2003) 